\title{
ON THE TRANSITION LAW OF TEMPERED STABLE ORNSTEIN-UHLENBECK PROCESSES
}

\author{
SHIBIN ZHANG, ${ }^{*}$ Shanghai Maritime University \\ XINSHENG ZHANG, ${ }^{* *}$ Fudan University
}

\begin{abstract}
In this paper, a stochastic integral of Ornstein-Uhlenbeck type is represented to be the sum of two independent random variables: one has a tempered stable distribution and the other has a compound Poisson distribution. In distribution, the compound Poisson random variable is equal to the sum of a Poisson-distributed number of positive random variables, which are independent and identically distributed and have a common specified density function. Based on the representation of the stochastic integral, we prove that the transition distribution of the tempered stable Ornstein-Uhlenbeck process is selfdecomposable and that the transition density is a $C^{\infty}$-function.
\end{abstract}

Keywords: Lévy process; tempered stable; Ornstein-Uhlenbeck-type process; selfdecomposability

2000 Mathematics Subject Classification: Primary 60J35

Secondary 62E15

\section{Introduction}

Recently, non-Gaussian processes of Ornstein-Uhlenbeck type have been widely used in describing stochastic volatility of finance assets (see [2]) and default intensities (see [4]). Therefore, the importance of the transition law of non-Gaussian processes of Ornstein-Uhlenbeck type is growing. For the general result of the transition law of these processes, see Lemma 17.1 of [9], where the characteristic function of the transition distribution is given. There is also some literature on the transition law of some important subclasses of these processes. For instance, the transition law of gamma OU processes is studied in [15], where the maximum likelihood estimator of the parameter is achieved by it; based on research on the transition law of inverse Gaussian Ornstein-Uhlenbeck (IGOU) processes, the exact simulation algorithm is obtained in [14].

The class of tempered stable Ornstein-Uhlenbeck (TSOU) processes is also an important subclass of non-Gaussian processes of Ornstein-Uhlenbeck type (see [3]). In this paper, the transition law of the TSOU process is determined by a stochastic integral of Ornstein-Uhlenbeck type. In [3], the stochastic integral of Ornstein-Uhlenbeck type is represented by an infinite series, so it is impossible from that to sample the innovations exactly for TSOU processes. For the same stochastic integral, the sample method based on the numeric inversion of the characteristic function (see [10]) is also approximate. However, the representation of the stochastic integral of Ornstein-Uhlenbeck type in this paper includes neither an infinite series

Received 29 August 2008; revision received 13 May 2009.

* Postal address: Department of Mathematics, Shanghai Maritime University, 1550 Pudong Avenue, Shanghai 200135,

P. R. China. Email address: sbzhang@shmtu.edu.cn

** Postal address: Department of Statistics, Fudan University, 220 Handan Road, Shanghai 200433, P. R. China.

Email address: xszhang@fudan.edu.cn 
nor a numeric approximation, so that it is more attractive to make parametric inference and exact simulation of TSOU processes. Moreover, we also obtain the self-decomposability of the distribution of the integral, and it is obvious that the self-decomposable distribution is of importance in theories and applications; see [2] and [9, Chapter 3].

The remainder of the paper is organized as follows. In Section 2 we provide some definitions and recall some properties of TSOU processes that we will need in order to develop the transition law and its properties. In Section 3 we set out the transition law of TSOU processes. In Section 4 we present some properties of the transition law. The paper closes with a discussion in Section 5.

\section{Preliminaries}

Firstly, we recall the definition of self-decomposability; for further details, the reader is referred to $[9$, p. 90].

Definition 2.1. A distribution $\mu$ on $\mathbb{R}$ is called self-decomposable if, for every $b>1$, there exists a distribution $\mu_{b}$ on $\mathbb{R}$ such that the characteristic functions $\hat{\mu}(z)$ and $\hat{\mu}_{b}(z)$ satisfy

$$
\hat{\mu}(z)=\hat{\mu}\left(b^{-1} z\right) \hat{\mu}_{b}(z), \quad z \in \mathbb{R} .
$$

A random variable with a self-decomposable distribution is called a self-decomposable random variable.

Alternatively, a random variable $X$ is self-decomposable if and only if, for each $b>1$, there exists a random variable $X_{b}$ which is independent of $X$ such that

$$
X \stackrel{\mathrm{D}}{=} b^{-1} X+X_{b}
$$

where ' $=$, denotes equality in distribution.

The following lemma is a special case of Corollary 15.11 of [9].

Lemma 2.1. Let $\mu$ be an infinitely divisible distribution on $(0,+\infty)$ with Lévy measure $v$. Then, $\mu$ is self-decomposable if and only if $v$ takes the special form

$$
v(\mathrm{~d} x)=\mathbf{1}_{\{x>0\}} \frac{k(x)}{x} \mathrm{~d} x,
$$

where $k(x) \geq 0, \int_{0}^{+\infty}\left(x^{2} \wedge 1\right)(k(x) / x) \mathrm{d} x<\infty$, and $k(x)$ is decreasing on $(0,+\infty)$.

The class of tempered stable (TS) distributions was introduced in [11]. Their applicability in survival analysis is discussed in [8].

Definition 2.2. Let $p(x ; \kappa, \delta)$ be the probability density function of the positively skewed $\kappa$-stable law $S(\kappa, \delta)$ with Laplace transform $\mathcal{L}(s)=\exp \left\{-\delta(2 s)^{\kappa}\right\}(\kappa \in(0,1)$ and $\delta>0)$. We call $\operatorname{TS}(\kappa, \delta, \gamma)$ a tempered stable distribution with parameters $\kappa \in(0,1), \delta>0$, and $\gamma \geq 0$ if its density is given by

$$
p(x ; \kappa, \delta, \gamma)=\mathrm{e}^{\delta \gamma} p(x ; \kappa, \delta) \mathrm{e}^{-\gamma^{1 / \kappa} x / 2} .
$$

That is, $p(x ; \kappa, \delta, \gamma)$ is the exponentially tilted version of $p(x ; \kappa, \delta)$.

For the $\operatorname{TS}(\kappa, \delta, \gamma)$ law, the parameter $\gamma$ controls the tail behavior. When $\gamma$ increases, we obtain a distribution with thinner tails. If $\kappa=\frac{1}{2}$, the $\operatorname{TS}(\kappa, \delta, \gamma)$ law becomes the inverse 
Gaussian (IG) law with parameters $\delta>0$ and $\gamma \geq 0$, denoted by $\operatorname{IG}(\delta, \gamma)$ (see [3]). The probability density function of the $\operatorname{IG}(\delta, \gamma)$ law has the closed form

$$
p(x ; \delta, \gamma)=\frac{\delta}{\sqrt{2 \pi}} \mathrm{e}^{\delta \gamma} x^{-3 / 2} \exp \left\{-\frac{1}{2}\left(\delta^{2} x^{-1}+\gamma^{2} x\right)\right\} \mathbf{1}_{\{x>0\}} .
$$

For general $\kappa \in(0,1)$, explicit expressions of $p(x ; \kappa, \delta)$ are known only in the form of series representations. Specifically, we have (cf., for instance, [7, p. 583])

$$
p(x, \kappa, \delta)=\frac{1}{2 \pi} \delta^{-1 / \kappa} \sum_{k=1}^{\infty} \frac{(-1)^{k-1}}{k !} \sin (k \kappa \pi) \Gamma(k \kappa+1) 2^{k \kappa+1}\left(x \delta^{-1 / \kappa}\right)^{-k \kappa-1} \mathbf{1}_{\{x>0\}} .
$$

The Lévy density of the $\operatorname{TS}(\kappa, \delta, \gamma)$ law (see [3]) is

$$
u(x)=\delta 2^{\kappa} \frac{\kappa}{\Gamma(1-\kappa)} x^{-1-\kappa} \exp \left\{-\frac{1}{2} \gamma^{1 / \kappa} x\right\}, \quad x>0 .
$$

Since $x u(x)$ is nonnegative and decreasing for $x>0$, it follows immediately from Lemma 2.1 that $\operatorname{TS}(\kappa, \delta, \gamma)$ is self-decomposable with support $[0, \infty)$. Since the class of self-decomposable distributions is a subclass of infinitely divisible distributions, the $\operatorname{TS}(\kappa, \delta, \gamma)$ distribution has the Laplace transformation (cf., for instance, [7, p. 450])

$$
\exp \left\{\int_{0}^{\infty}\left(\mathrm{e}^{-u x}-1\right) \delta 2^{\kappa} \frac{\kappa}{\Gamma(1-\kappa)} x^{-1-\kappa} \exp \left\{-\frac{1}{2} \gamma^{1 / \kappa} x\right\} \mathrm{d} x\right\} .
$$

Extending this expression to the left-hand half-plane $\{w \in \mathbb{C}: \operatorname{Re} w \leq 0\}$ by analyticity inside and continuity to the boundary, and letting $w=\mathrm{i} z, z \in \mathbb{R}$, we obtain the characteristic function of the $\operatorname{TS}(\kappa, \delta, \gamma)$ law,

$$
\psi(z)=\exp \left\{\int_{0}^{\infty}\left(\mathrm{e}^{\mathrm{i} z x}-1\right) \delta 2^{\kappa} \frac{\kappa}{\Gamma(1-\kappa)} x^{-1-\kappa} \exp \left\{-\frac{1}{2} \gamma^{1 / \kappa} x\right\} \mathrm{d} x\right\} .
$$

Given a $\operatorname{TS}(\kappa, \delta, \gamma)$ law, it follows from, for instance, [2] (and the references therein) that there exists a stationary process $X(t)$ and a Lévy process $Z(t)$, independent of $X(0)$, such that $X(t) \sim \operatorname{TS}(\kappa, \delta, \gamma)$ and, for all $\lambda>0, X(t)$ solves the following stochastic differential equation of Ornstein-Uhlenbeck type:

$$
\mathrm{d} X(t)=-\lambda X(t) \mathrm{d} t+\mathrm{d} Z(\lambda t), \quad \lambda>0 .
$$

It is easily verified that a (strong) solution to the Ornstein-Uhlenbeck equation, (2.4), is given by

$$
X(t)=\mathrm{e}^{-\lambda t} X(0)+\int_{(0, t]} \mathrm{e}^{-\lambda(t-s)} \mathrm{d} Z(\lambda s) .
$$

Up to indistinguishability, this solution is unique (see [9, p. 104]). Since the marginal distribution of the stationary process $X(t)$ is TS and $X(t)$ satisfies (2.4), the process $X(t)$ is called a TSOU process (see, for instance, [3]). As $Z(t)$ is used to drive the Ornstein-Uhlenbeck process, it is referred to as the background driving Lévy process. 
The law of the stochastic integral $\int_{(0, t]} \mathrm{e}^{-\lambda(t-s)} \mathrm{d} Z(\lambda s)$ plays an important role in finding the transition law of the TSOU process $X(t)$. In [3], this integral is represented as

$$
\int_{(0, t]} \mathrm{e}^{-\lambda(t-s)} \mathrm{d} Z(\lambda s) \stackrel{\mathrm{D}}{=} \sum_{i=1}^{\infty} \exp \left\{-\lambda t r_{i}\right\} \min \left\{\left(\frac{a_{i} \kappa}{A \lambda t}\right)^{-1 / \kappa}, e_{i} v_{i}^{1 / \kappa}\right\}+\sum_{i=1}^{N(\lambda t)} \exp \left\{-\lambda t r_{i}^{*}\right\} c_{i}
$$

where $\left\{e_{i}\right\},\left\{v_{i}\right\},\left\{a_{i}\right\},\left\{r_{i}\right\},\left\{r_{i}^{*}\right\}$, and $\left\{c_{i}\right\}$ are independent of one another and over $i$, except for the $\left\{a_{i}\right\}$ process. Here the $\left\{e_{i}\right\}$ are exponential with mean $1 / B,\left\{v_{i}\right\},\left\{r_{i}\right\}$, and $\left\{r_{i}^{*}\right\}$ are independent and identically distributed standard uniforms, the $\left\{c_{i}\right\}$ are independent and identically distributed $\Gamma\left(1-\kappa, \frac{1}{2} \gamma^{1 / \kappa}\right) \mathrm{s}$, and $A=\delta 2^{\kappa} \kappa^{2} / \Gamma(1-\kappa)$ and $B=\frac{1}{2} \gamma^{1 / \kappa}$. Furthermore, the $a_{1}<a_{2}<\cdots<a_{i}<\cdots$ are arrival times of a Poisson process with intensity 1 , while $N(t)$ is a Poisson process with intensity $\delta \gamma \kappa$. Equality (2.5) includes an infinite series representation, so it is impossible from that to sample the innovations exactly for TSOU processes. For the same stochastic integral, the sample method based on the numeric inversion of the characteristic function (see [10]) is also approximate.

\section{The transition law of TSOU processes}

Since $X(t) \sim \operatorname{TS}(\kappa, \delta, \gamma)$, its characteristic function is $\psi(z)$, defined in (2.3). Let $\phi(z)$ denote the characteristic function of $Z(1)$. As noted Remark 4.3 of [1], $\phi(x)$ and $\psi(x)$ are related by

$$
\log \phi(z)=z \frac{\partial \log \psi(z)}{\partial z}
$$

Hence, the characteristic function of $Z(1)$ is

$$
\phi(z)=\exp \left\{\int_{0}^{\infty}\left(\mathrm{e}^{\mathrm{i} z x}-1\right) v(x) \mathrm{d} x\right\}
$$

where

$$
v(x)=\delta 2^{\kappa} \frac{\kappa}{\Gamma(1-\kappa)}\left(\kappa x^{-1}+\frac{1}{2} \gamma^{1 / \kappa}\right) x^{-\kappa} \exp \left\{-\frac{1}{2} \gamma^{1 / \kappa} x\right\} .
$$

Let $P_{t}(x, B)$ denote the temporally homogeneous transition function of the TSOU process $X(t)$ over a time interval of length $t$, i.e. the conditional probability $\mathrm{P}(X(t) \in B \mid X(0)=x)$, where $B$ is a Borel measurable subset of $\mathbb{R}$. By Lemma 17.1 of [9], the temporally homogeneous transition function $P_{t}(x, B)$ satisfies

$$
\int_{-\infty}^{\infty} \mathrm{e}^{\mathrm{i} z y} P_{t}(x, \mathrm{~d} y)=\exp \left\{\mathrm{i} z \mathrm{e}^{-\lambda t} x+\lambda \int_{0}^{t} \varphi\left(\mathrm{e}^{-\lambda(t-s)} z\right) \mathrm{d} s\right\}
$$

where $\varphi(z)=\log (\phi(z))$ and $\phi(z)$ is the characteristic function of $Z(1)$.

Theorem 3.1. If $\gamma>0$, the TSOU process $X(t)$, given that $X(0)=x$, can be represented as the sum of a constant, a TS random variable, and a compound Poisson random variable in distribution, that is,

$$
\left.X(t)\right|_{X(0)=x} \stackrel{\mathrm{D}}{=} \mathrm{e}^{-\lambda t} x+W_{0}^{t}+\sum_{i=1}^{\tilde{N}^{t}} W_{i}^{t},
$$


where $W_{0}^{t} \sim \operatorname{TS}\left(\kappa, \delta\left(1-\mathrm{e}^{-\kappa \lambda t}\right), \gamma\right)$, the random variable $\tilde{N}^{t}$ has a Poisson distribution of intensity $\delta \gamma\left(1-\mathrm{e}^{-\kappa \lambda t}\right)$, and $W_{1}^{t}, W_{2}^{t}, \ldots$ are independent random variables having a common specified density function,

$$
f_{W^{t}}(w)=\frac{2^{\kappa} \kappa \gamma^{-1}}{\Gamma(1-\kappa)}\left(\mathrm{e}^{\kappa \lambda t}-1\right)^{-1} w^{-\kappa-1}\left(\exp \left\{-\frac{1}{2} \gamma^{1 / \kappa} w\right\}-\exp \left\{-\frac{1}{2} \gamma^{1 / \kappa} w \mathrm{e}^{\lambda t}\right\}\right) \mathbf{1}_{\{w>0\}} .
$$

Furthermore, $\left\{W_{0}^{t}\right\},\left\{W_{1}^{t}, W_{2}^{t}, \ldots\right\}$, and $\left\{\tilde{N}^{t}\right\}$ are independent of one another.

Proof. For the TSOU process $X(t)$, the characteristic function of $Z(1)$ is defined in (3.1), so that, by (3.3),

$$
\begin{aligned}
\int_{-\infty}^{\infty} \mathrm{e}^{\mathrm{i} z y} P_{t}(x, \mathrm{~d} y) & =\exp \left\{\mathrm{i} z \mathrm{e}^{-\lambda t} x+\lambda \int_{0}^{t}\left(\int_{0}^{\infty}\left(\exp \left\{\mathrm{ie}^{-\lambda(t-s)} z x\right\}-1\right) v(x) \mathrm{d} x\right) \mathrm{d} s\right\} \\
& =\exp \left\{\mathrm{i} z \mathrm{e}^{-\lambda t} x+\lambda \int_{0}^{t}\left(\int_{0}^{\infty}\left(\exp \left\{\mathrm{ie}^{-\lambda u} z x\right\}-1\right) v(x) \mathrm{d} x\right) \mathrm{d} u\right\} \\
& =\exp \left\{\mathrm{i} z \mathrm{e}^{-\lambda t} x+\lambda \int_{0}^{\infty} v(x)\left(\int_{0}^{t}\left(\exp \left\{\mathrm{ie}^{-\lambda u} z x\right\}-1\right) \mathrm{d} u\right) \mathrm{d} x\right\} \\
& =\exp \left\{\mathrm{i} z \mathrm{e}^{-\lambda t} x+\int_{0}^{\infty} v(x)\left(\int_{\mathrm{e}^{-\lambda t} x}^{x}\left(\mathrm{e}^{\mathrm{i} z w}-1\right) \frac{1}{w} \mathrm{~d} w\right) \mathrm{d} x\right\} \\
& =\exp \left\{\mathrm{i} z \mathrm{e}^{-\lambda t} x+\int_{0}^{\infty}\left(\mathrm{e}^{\mathrm{i} z w}-1\right) \frac{\int_{w}^{\mathrm{e}^{\lambda t} w} v(x) \mathrm{d} x}{w} \mathrm{~d} w\right\}
\end{aligned}
$$

where $v(x)$ is defined in (3.2). Here we have adopted the transformations $u=t-s$ and $w=\mathrm{e}^{-\lambda u} x$ respectively in the second and fourth equalities. Let $k_{t}(w)=\int_{w}^{\mathrm{e}^{\lambda t} w} v(x) \mathrm{d} x$. Then

$$
\begin{aligned}
k_{t}(w) & =\frac{\delta 2^{\kappa} \kappa}{\Gamma(1-\kappa)} \int_{w}^{\mathrm{e}^{\lambda t} w}\left(\kappa x^{-1}+\frac{1}{2} \gamma^{1 / \kappa}\right) x^{-\kappa} \exp \left\{-\frac{1}{2} \gamma^{1 / \kappa} x\right\} \mathrm{d} x \\
& =\frac{\delta 2^{\kappa-1} \kappa}{\Gamma(1-\kappa)} w^{-\kappa} \int_{0}^{\lambda t}\left(2 \kappa \mathrm{e}^{-\kappa h}+\gamma^{1 / \kappa} \mathrm{e}^{(1-\kappa) h} w\right) \exp \left\{-\frac{1}{2} \gamma^{1 / \kappa} w \mathrm{e}^{h}\right\} \mathrm{d} h \\
& =\frac{\delta 2^{\kappa-1} \kappa}{\Gamma(1-\kappa)} w^{-\kappa} \int_{0}^{\lambda t} \mathrm{~d}\left(-2 \mathrm{e}^{-\kappa h} \exp \left\{-\frac{1}{2} \gamma^{1 / \kappa} w \mathrm{e}^{h}\right\}\right) \\
& =\frac{\delta 2^{\kappa} \kappa}{\Gamma(1-\kappa)} w^{-\kappa}\left(\exp \left\{-\frac{1}{2} \gamma^{1 / \kappa} w\right\}-\mathrm{e}^{-\kappa \lambda t} \exp \left\{-\frac{1}{2} \gamma^{1 / \kappa} w \mathrm{e}^{\lambda t}\right\}\right),
\end{aligned}
$$

where the second equality follows from the transformation $h=\ln x-\ln w$. Hence

$$
\begin{aligned}
\int_{-\infty}^{\infty} \mathrm{e}^{\mathrm{i} z y} P_{t}(x, \mathrm{~d} y) & =\exp \left\{\mathrm{i} z \mathrm{e}^{-\lambda t} x+\int_{0}^{\infty}\left(\mathrm{e}^{\mathrm{i} z w}-1\right) \frac{k_{t}(w)}{w} \mathrm{~d} w\right\} \\
& =\exp \left\{\mathrm{i}^{-\lambda} \mathrm{e}^{-\lambda t} x\right\} \phi_{1}(z) \phi_{2}(z),
\end{aligned}
$$

where

$$
\begin{aligned}
& \phi_{1}(z)=\exp \left\{\int_{0}^{\infty}\left(\mathrm{e}^{\mathrm{i} z w}-1\right) \frac{\delta\left(1-\mathrm{e}^{-\kappa \lambda t}\right) 2^{\kappa} \kappa}{\Gamma(1-\kappa)} w^{-\kappa-1} \exp \left\{-\frac{1}{2} \gamma^{1 / \kappa} w\right\} \mathrm{d} w\right\}, \\
& \phi_{2}(z)=\exp \left\{\int_{0}^{\infty} \delta \gamma\left(1-\mathrm{e}^{-\kappa \lambda t}\right)\left(\mathrm{e}^{\mathrm{i} z w}-1\right) f_{W^{t}}(w) \mathrm{d} w\right\}
\end{aligned}
$$


and $f_{W^{t}}(w)$ is as defined in (3.5). That $\phi_{1}(z)$ is the characteristic function of the $\operatorname{TS}(\kappa, \delta(1-$ $\left.\mathrm{e}^{-\kappa \lambda t}\right), \gamma$ ) law can be shown by (2.3). Hence, $\phi_{1}(z)$ can be treated as the characteristic function of the distribution of the random variable $W_{0}^{t}$ described in (3.4). For $w>0, f_{W^{t}}(w) \geq 0$, and

$$
\int_{0}^{\infty} f_{W^{t}}(w) \mathrm{d} w=\frac{2^{\kappa} \kappa \gamma^{-1}}{\Gamma(1-\kappa)}\left(\mathrm{e}^{\kappa \lambda t}-1\right)^{-1}\left(\frac{\gamma}{2^{\kappa}}-\frac{\gamma \mathrm{e}^{\kappa \lambda t}}{2^{\kappa}}\right) \Gamma(-\kappa)=1,
$$

we have shown that $f_{W^{t}}(w)$ defined in (3.5) is a density function. Hence, $\phi_{2}(z)$ is a characteristic function of a compound Poisson distribution. The compound Poisson distribution is the same as the distribution of the random variable $\sum_{i=1}^{N^{t}} W_{i}^{t}$ described in (3.4). Equalities (3.7)-(3.8) imply that $\left\{W_{0}^{t}\right\},\left\{W_{1}^{t}, W_{2}^{t}, \ldots\right\}$, and $\left\{\tilde{N}^{t}\right\}$ are independent of one another. This completes the proof.

Remark 3.1. If $\kappa=\frac{1}{2}$, the marginal distribution of the TSOU process $X(t)$ becomes the IG distribution with parameters $\delta>0$ and $\gamma \geq 0$, i.e. $X(t) \sim \operatorname{IG}(\delta, \gamma)$. Now, the marginal density of $X(t)$ in (2.1) has the closed form (2.2). The process $\{X(t), t \geq 0\}$ is referred to as an IGOU process (see [3]). Here, if $\gamma>0, W_{i}^{t}(i=1,2, \ldots)$ in (3.4) has the simpler probability density function

$$
f_{W^{t}}(w)=\frac{\gamma^{-1}}{\sqrt{2 \pi}}\left(\mathrm{e}^{\lambda t / 2}-1\right)^{-1} w^{-3 / 2}\left(\exp \left\{-\frac{1}{2} \gamma^{2} w\right\}-\exp \left\{-\frac{1}{2} \gamma^{2} w \mathrm{e}^{\lambda t}\right\}\right) \mathbf{1}_{\{w>0\}} .
$$

In terms of the transition law of the IGOU process, the exact simulation algorithm of the process is obtained in [14].

Remark 3.2. Based on Theorem 3.1, the exact simulation method of the TSOU process $X(t)$ can be implemented, which is more reliable than the approximate method based on Rosinski's series representation, (2.5). The implementation of an exact simulation of the TSOU process is achievable for the following two main reasons.

1. If $X \sim S(\kappa, \delta)$ then $X$ has decomposition (a special case of the result in [5] or [12]; see also [13])

$$
X \stackrel{\mathrm{D}}{=} 2\left(\delta \cos \left(\frac{\kappa \pi}{2}\right)\right)^{1 / \kappa} \frac{\sin \kappa(\pi / 2+U)}{(\cos (U))^{1 / \kappa}}\left(\frac{\cos (U-\kappa(\pi / 2+U))}{E}\right)^{1 / \kappa-1},
$$

where $U$ and $E$ are independent of each other, $U$ is uniformly distributed on $(-\pi / 2, \pi / 2)$, and $E$ is exponential with mean 1. Furthermore, by equality (2.1),

$$
p(x ; \kappa, \delta, \gamma) \leq \mathrm{e}^{\delta \gamma} p(x ; \kappa, \delta) \text { for } x>0
$$

and $\mathrm{e}^{\delta \gamma}$ is a known constant. Hence, the generation of the $\operatorname{TS}(\kappa, \delta, \gamma)$ random variate can be implemented by the acceptance-rejection sampling technique.

2. The generation of $W_{i}^{t}(i=1,2, \ldots)$ in Theorem 3.1 can also be implemented by the acceptance-rejection sampling technique. For $w>0$,

$$
\begin{aligned}
\exp \left\{-\frac{1}{2} \gamma^{1 / \kappa} w\right\}-\exp \left\{-\frac{1}{2} \gamma^{1 / \kappa} w \mathrm{e}^{\lambda t}\right\} & =\frac{1}{2} \gamma^{1 / \kappa} \int_{w}^{w \mathrm{e}^{\lambda t}} \exp \left\{-\frac{1}{2} \gamma^{1 / \kappa} x\right\} \mathrm{d} x \\
& \leq \frac{1}{2} \gamma^{1 / \kappa} \exp \left\{-\frac{1}{2} \gamma^{1 / \kappa} w\right\} w\left(\mathrm{e}^{\lambda t}-1\right)
\end{aligned}
$$


so that $f_{W^{t}}(w)$ defined in (3.5) satisfies

$$
f_{W^{t}}(w) \leq M \frac{\left(\gamma^{1 / \kappa} / 2\right)^{1-\kappa}}{\Gamma(1-\kappa)} w^{-\kappa} \exp \left\{-\frac{1}{2} \gamma^{1 / \kappa} w\right\},
$$

where $M>0$ is a known constant and $\left(\left(\gamma^{1 / \kappa} / 2\right)^{1-\kappa} / \Gamma(1-\kappa)\right) w^{-\kappa} \exp \left\{-\frac{1}{2} \gamma^{1 / \kappa} w\right\}$ is the density of the $\Gamma\left(1-\kappa, \frac{1}{2} \gamma^{1 / \kappa}\right)$ distribution. Thus, the acceptance-rejection sampling technique can be employed. Furthermore, combining the acceptance-rejection method with the composition method (see [6, p. 66]), we find a method to improve the acceptance probability as close to 1 as possible. The method used to improve the acceptance probability is the same as that in [14].

Theorem 3.2. If $\gamma>0$, the TSOU process $X(t)$, given that $X(0)=x$, can be represented as the sum of a constant, a TS random variable, and a compound Poisson random variable in distribution, that is,

$$
X(t) \mid X(0)=x \stackrel{\mathrm{D}}{=} \mathrm{e}^{-\lambda t} x+\bar{W}_{0}^{t}+\sum_{i=1}^{\bar{N}^{t}} \bar{W}_{i}^{t},
$$

where $\bar{W}_{0}^{t} \sim \operatorname{TS}\left(\kappa, \delta\left(1-\mathrm{e}^{-\kappa \lambda t}\right), \gamma \mathrm{e}^{\kappa \lambda t}\right)$, the random variable $\bar{N}^{t}$ has a Poisson distribution of intensity $\delta \gamma\left(\mathrm{e}^{\kappa \lambda t}-1\right)$, and $\bar{W}_{1}^{t}, \bar{W}_{2}^{t}, \ldots$ are independent random variables having a common specified density function $f_{W^{t}}(w)$ defined in (3.5). Furthermore, $\left\{\bar{W}_{0}^{t}\right\},\left\{\bar{W}_{1}^{t}, \bar{W}_{2}^{t}, \ldots\right\}$, and $\left\{\bar{N}^{t}\right\}$ are independent of one another.

Proof. Representation (3.7) has the alternative form

$$
\begin{aligned}
\int_{-\infty}^{\infty} \mathrm{e}^{\mathrm{i} z y} P_{t}(x, \mathrm{~d} y) & =\exp \left\{\mathrm{i} z \mathrm{e}^{-\lambda t} x+\int_{0}^{\infty}\left(\mathrm{e}^{\mathrm{i} z w}-1\right) \frac{k_{t}(w)}{w} \mathrm{~d} w\right\} \\
& =\exp \left\{\mathrm{i} z \mathrm{e}^{-\lambda t} x\right\} \bar{\phi}_{1}(z) \bar{\phi}_{2}(z),
\end{aligned}
$$

where

$$
\begin{aligned}
& \bar{\phi}_{1}(z)=\exp \left\{\int_{0}^{\infty}\left(\mathrm{e}^{\mathrm{i} z w}-1\right) \frac{\delta\left(1-\mathrm{e}^{-\kappa \lambda t}\right) 2^{\kappa} \kappa}{\Gamma(1-\kappa)} w^{-\kappa-1} \exp \left\{-\frac{1}{2}\left(\gamma \mathrm{e}^{\kappa \lambda t}\right)^{1 / \kappa} w\right\} \mathrm{d} w\right\}, \\
& \bar{\phi}_{2}(z)=\exp \left\{\int_{0}^{\infty} \delta \gamma\left(\mathrm{e}^{\kappa \lambda t}-1\right)\left(\mathrm{e}^{\mathrm{i} z w}-1\right) f_{W^{t}}(w) \mathrm{d} w\right\},
\end{aligned}
$$

and $f_{W^{t}}(w)$ is as defined in (3.5). For the same reason as in the proof of Theorem 3.1, $\bar{\phi}_{1}(z)$ is the characteristic function of the distribution of the random variable $\bar{W}_{0}^{t}$ and $\bar{\phi}_{2}(z)$ is a characteristic function of a compound Poisson distribution of the random variable $\sum_{i=1}^{N^{t}} \bar{W}_{i}^{t}$. Here, both $\bar{W}_{0}^{t}$ and $\sum_{i=1}^{\bar{N}^{t}} \bar{W}_{i}^{t}$ are described in (3.10).

Since the probability density function of the $\operatorname{TS}(\kappa, \delta, \gamma)$ law is the exponentially tilted version of that of the $S(\kappa, \delta)$ law, the latter can be treated as a special case of the $\operatorname{TS}(\kappa, \delta, \gamma)$ law. If $\gamma=0$, it follows from (2.3) that the characteristic function of the $S(\kappa, \delta)$ law is

$$
\psi(z)=\exp \left\{\int_{0}^{\infty}\left(\mathrm{e}^{\mathrm{i} z x}-1\right) \delta 2^{\kappa} \frac{\kappa}{\Gamma(1-\kappa)} x^{-1-\kappa} \mathrm{d} x\right\} .
$$

If $\gamma=0$, the marginal distribution of the $\operatorname{TS}(\kappa, \delta, \gamma)$ OU process becomes the $S(\kappa, \delta)$ law, so that we refer to this process as the positively skewed stable OU process. 
Corollary 3.1. The positively skewed stable OU process $X(t)$, given that $X(0)=x$, can be represented as

$$
\left.X(t)\right|_{X(0)=x} \stackrel{\mathrm{D}}{=} \mathrm{e}^{-\lambda t} x+W_{0}^{t}
$$

where $W_{0}^{t} \sim S\left(\kappa, \delta\left(1-\mathrm{e}^{-\kappa \lambda t}\right)\right)$.

Proof. By equality (3.6), if $\gamma=0$ then

$$
k_{t}(w)=\delta\left(1-\mathrm{e}^{-\kappa \lambda t}\right) 2^{\kappa} \frac{\kappa}{\Gamma(1-\kappa)} w^{-\kappa} .
$$

Hence, the temporally homogeneous transition function $P_{t}(x, B)$ satisfies

$$
\int_{-\infty}^{\infty} \mathrm{e}^{\mathrm{i} z y} P_{t}(x, \mathrm{~d} y)=\exp \left\{\mathrm{i} z \mathrm{e}^{-\lambda t} x\right\} \varphi(z)
$$

where

$$
\varphi(z)=\exp \left\{\int_{0}^{\infty}\left(\mathrm{e}^{\mathrm{i} z w}-1\right) \delta\left(1-\mathrm{e}^{-\kappa \lambda t}\right) 2^{\kappa} \frac{\kappa}{\Gamma(1-\kappa)} w^{-\kappa-1} \mathrm{~d} w\right\} .
$$

It follows from equality (3.11) that $\varphi(z)$ is the characteristic function of the $S\left(\kappa, \delta\left(1-\mathrm{e}^{-\kappa \lambda t}\right)\right)$ law. This completes the proof.

Remark 3.3. For the positively skewed $\kappa$-stable random variable, since we have decomposition (3.9), the generation of $W_{0}^{t}$ in Corollary 3.1 can be exactly implemented. Thus, exact simulation of the positively skewed stable OU process $X(t)$ can also be easily implemented by (3.12).

\section{Some properties of the transition law}

The function $k_{t}(w)$ defined by (3.6) has the following properties.

Lemma 4.1. If $\frac{1}{2} \leq \kappa<1$ and $\gamma>0$, then, for $w>0, k_{t}(w) \geq 0$,

$$
\int_{0}^{+\infty}\left(w^{2} \wedge 1\right) \frac{k_{t}(w)}{w} \mathrm{~d} x<\infty
$$

and $k_{t}(w)$ is decreasing on $(0, \infty)$.

Proof. For $w>0, k_{t}(w) \geq 0$ is obvious. We have $\int_{0}^{\infty}\left(w^{2} \wedge 1\right)\left(k_{t}(w) / w\right) \mathrm{d} w<\infty$ because of

$$
\int_{0}^{1} w k_{t}(w) \mathrm{d} w \leq \frac{\delta 2^{\kappa} \kappa}{\Gamma(1-\kappa)} \int_{0}^{1} w^{1-\kappa} \exp \left\{-\frac{1}{2} \gamma^{1 / \kappa} w\right\} \mathrm{d} w \leq \frac{\delta 2^{\kappa} \kappa}{\Gamma(1-\kappa)}<\infty
$$

and

$$
\begin{aligned}
\int_{1}^{\infty} \frac{k_{t}(w)}{w} \mathrm{~d} w & \leq \frac{\delta 2^{\kappa} \kappa}{\Gamma(1-\kappa)} \int_{1}^{\infty} w^{-\kappa-1} \exp \left\{-\frac{1}{2} \gamma^{1 / \kappa} w\right\} \mathrm{d} w \\
& \leq \frac{\delta 2^{\kappa} \kappa}{\Gamma(1-\kappa)} \int_{1}^{\infty} \exp \left\{-\frac{1}{2} \gamma^{1 / \kappa} w\right\} \mathrm{d} w \\
& <\infty
\end{aligned}
$$


If $\kappa, \delta, \gamma, \lambda$, and $t$ are fixed, then $k_{t}(w)$ is a function of $w$ only. We have

$$
\begin{aligned}
\frac{\mathrm{d} k_{t}(w)}{\mathrm{d} w}=\frac{\delta 2^{\kappa} \kappa}{\Gamma(1-\kappa)} w^{-\kappa-1} & {\left[\left(\kappa+\frac{1}{2} \gamma^{1 / \kappa} w \mathrm{e}^{\lambda t}\right) \mathrm{e}^{-\kappa \lambda t} \exp \left\{-\frac{1}{2} \gamma^{1 / \kappa} w \mathrm{e}^{\lambda t}\right\}\right.} \\
& \left.-\left(\kappa+\frac{1}{2} \gamma^{1 / \kappa} w\right) \exp \left\{-\frac{1}{2} \gamma^{1 / \kappa} w\right\}\right] .
\end{aligned}
$$

For fixed $0<\kappa<1, w>0$, and $\gamma \geq 0$, we consider the function

$$
f(x)=\left(\kappa+\frac{1}{2} \gamma^{1 / \kappa} w x\right) x^{-\kappa} \exp \left\{-\frac{1}{2} \gamma^{1 / \kappa} w x\right\},
$$

where $1 \leq x \leq \mathrm{e}^{\lambda t}$. If $\frac{1}{2} \leq \kappa<1$ then $f(x)$ is a monotone decreasing function for $1 \leq x \leq \mathrm{e}^{\lambda t}$ because

$$
\begin{aligned}
f^{\prime}(x)= & \left(\frac{1}{2}-\kappa\right) \gamma^{1 / \kappa} w x^{-\kappa} \exp \left\{-\frac{1}{2} \gamma^{1 / \kappa} w x\right\} \\
& -\left(\kappa^{2} x^{-1-\kappa}+\frac{1}{4} \gamma^{2 / \kappa} w^{2} x^{1-\kappa}\right) \exp \left\{-\frac{1}{2} \gamma^{1 / \kappa} w x\right\} \\
< & 0 .
\end{aligned}
$$

Now, for $w>0$,

$$
\frac{\mathrm{d} k_{t}(w)}{\mathrm{d} w}=\frac{\delta 2^{\kappa} \kappa}{\Gamma(1-\kappa)} w^{-\kappa-1}\left[f\left(\mathrm{e}^{\lambda t}\right)-f(1)\right] \leq 0,
$$

where $f$ is defined in (4.1). So $k_{t}(w)$ is decreasing on $(0, \infty)$.

Lemma 4.2. If $\gamma=0$ then, for $w>0, k_{t}(w) \geq 0, \int_{0}^{+\infty}\left(w^{2} \wedge 1\right)\left(k_{t}(w) / w\right) \mathrm{d} x<\infty$, and $k_{t}(w)$ is decreasing on $(0, \infty)$.

Proof. The proof is the same as that of Lemma 4.1.

Theorem 4.1. If $\frac{1}{2} \leq \kappa<1$ and $\gamma>0$ or $0<\kappa<1$ and $\gamma=0$, then the transition law of the TSOU process $X(t)$ has the following properties.

1. The transition law of the TSOU process $X(t)$ is self-decomposable.

2. For fixed $t>0$ and $X(0)=x>0$, the transition law of the TSOU process $X(t)$ has a density with respect to Lebesgue measure. Here the transition density is denoted $p(t, x, y)$.

3. For fixed $t>0$ and $x>0$, the transition density $p(t, x, y)$ is a $C^{\infty}$-function with respect to $y$ on $\mathbb{R}$.

Proof. Since the Lévy measure of the transition law has the form

$$
v_{t}(w)=\frac{k_{t}(w)}{w} \mathrm{~d} w
$$

where $k_{t}(w)$ is as defined in (3.6), the self-decomposability of the transition law of $X(t)$ follows from Lemma 2.1, Lemma 4.1, and Lemma 4.2. By Theorem 27.13 of [9], for fixed $t>0$ and $X(0)=x>0$, the transition function $P_{t}(x, y)$ of the process $X(t)$ is absolutely continuous with respect to $y$ on $\mathbb{R}$. We denote the density by $p(t, x, y)$. Since $k_{t}(0+)=\lim _{w \rightarrow 0+} k_{t}(w)=+\infty$, by Theorem 28.4 of [9], the process $X(t)$ has a $C^{\infty}$ transition density with respect to $y$ on $\mathbb{R}$. 


\section{Discussion}

In this paper, the transition law of the TSOU process is represented as (3.4), by which exact simulation of the process can be implemented. Otherwise, based on the infinite series representation, (2.5), exact simulation is impossible. The error of the approximate simulation method based on (2.5) is influenced by the value of the parameter of the process (see [14]). Since the $S(\kappa, \delta)$ OU process can be treated as a special case of the $\operatorname{TS}(\kappa, \delta, \gamma)$ OU process when $\gamma$ is equal to 0 , representation (3.12) can also be treated as a special case of (3.4) when $\gamma$ is equal to 0 . If $\gamma=0, W_{0}^{t} \sim \operatorname{TS}\left(\kappa, \delta\left(1-\mathrm{e}^{-\kappa \lambda t}\right), \gamma\right)$ and $\sum_{i=1}^{N^{t}} W_{i}^{t}$ in (3.4) respectively degenerate into $W_{0}^{t} \sim S\left(\kappa, \delta\left(1-\mathrm{e}^{-\kappa \lambda t}\right)\right)$ and 0 . But, if $\gamma=0$, representation (2.5) is invalid.

The class of self-decomposable distributions is a subclass of the class of infinitely divisible distributions. Its importance in the theory of Lévy processes, processes of OrnsteinUhlenbeck type, and self-similar additive processes, and in applications, is growing. See [2] and [9, Chapter 3]. In this paper, the law of the stochastic integral $\int_{(0, t]} \mathrm{e}^{-\lambda(t-s)} \mathrm{d} Z(\lambda s)$ is self-decomposable. It belongs to a new four-parameter distribution having the following characteristic function:

$\psi(z)=\exp \left\{\int_{0}^{\infty}\left(\mathrm{e}^{\mathrm{i} z x}-1\right) \frac{\delta 2^{\kappa} \kappa}{\Gamma(1-\kappa)} x^{-\kappa-1}\left(\exp \left\{-\frac{1}{2} \gamma^{1 / \kappa} x\right\}-\zeta \exp \left\{-\frac{1}{2}\left(\frac{\gamma}{\zeta}\right)^{1 / \kappa} x\right\}\right) \mathrm{d} x\right\}$,

where $\kappa \in\left[\frac{1}{2}, 1\right), \zeta \in(0,1), \delta>0$, and $\gamma \geq 0$ are parameters. The $\operatorname{TS}(\kappa, \delta, \gamma)$ law can be treated as the special case as $\zeta \rightarrow 0+$. It is also an interesting problem to see properties of this new four-parameter distribution.

\section{Acknowledgements}

This research was supported by the Natural Science Foundation of China (grant number 10671037), the Natural Science Foundation of China (grant number 10901100), and the Science Foundation of Shanghai Educational Committee (grant number 06FZ035).

\section{References}

[1] Barndorff-Nielsen, O. E. And Shephard, N. (2001). Modelling by Lévy processes for financial econometrics. In Lévy Processes, eds O. E. Barndorff-Nielsen, T. Mikosch and S. Resnick, Birkhäuser, Boston, MA, pp. 283-318.

[2] BarndorfF-Nielsen, O. E. And Shephard, N. (2001). Non-Gaussian Ornstein-Uhlenbeck-based models and some of their uses in financial economics (with discussion). J. R. Statist. Soc. B 63, 167-241.

[3] Barndorff-Nielsen, O. E. And Shephard, N. (2001). Normal modified stable processes. Theory Prob. Math. Statist. 65, 1-20.

[4] CARiboni, C. And Schoutens, W. (2009). Jumps in intensity models: investigating the performance of OrnsteinUhlenbeck processes in credit risk modeling. Metrika 69, 173-198.

[5] Chambers, J. M., Mallows, C. L. and StucK, B.W. (1976). A method for simulating stable random variables. J. Amer. Statist. Assoc. 71, 340-344.

[6] Devroye, L. (1986). Nonuniform Random Variate Generation. Springer, New York.

[7] Feller, W. (1971). An Introduction to Probability Theory and Its Applications, Vol. II, 2nd edn. John Wiley, New York.

[8] Hougande, P. (1986). Survival models for heterogeneous populations derived from stable distributions. Biometrika 73, 387-396.

[9] Sato, K. I. (1999). Lévy Processes and Infinitely Divisible Distributions. Cambridge University Press.

[10] Taufer, E. And Leonenko, N. (2009). Simulation of Lévy-driven Ornstein-Uhlenbeck processes with given marginal distribution. Comput. Statist. Data Anal. 53, 2427-2437.

[11] Tweedie, M. C. K. (1984). An index which distinguishes between some important exponential families. In Statistics: Applications and New Directions, eds J. Ghosh and J. Roy, Proceedings of the Indian Statistical Institute Golden Jubilee International Conference, pp. 579-604. 
[12] Weron, R. (1996). On the Chambers-Mallows-Stuck method for simulating skewed stable random variables. Statist. Prob. Lett. 28, 165-171.

[13] Zhang, S. (2008). Simulation of non-Gaussian OU-based stochastic volatility models. In Proc. Internat. Symp. Financial Eng. Risk Manag., eds C. Ai and D. Wu, Universe Academic Press, Toronto, pp. 234-238.

[14] Zhang, S. And Zhang, X. (2008). Exact simulation of IG-OU processes. Methodology Comput. Appl. Prob. 10, 337-355.

[15] Zhang, S., Zhang, X. And Sun, S. (2006). Parametric estimation of discretly sampled Gamma-OU processes. Science China Ser. A 49, 1231-1257. 